\title{
Supply Chain Management in Agile Manufacturing Environment
}

\author{
Toshiya Kaihara
}

Dept. of Computer and Systems Engineering, Faculty of Engineering, Kobe University

E-mail: kaihara@ms.cs.kobe-u.ac.jp

\begin{abstract}
Supply chain management (SCM) is now recognised as one of the best means by which enterprises can make instant improvements to their business strategies and operations. Computational virtual market based supply chain operation solves the product allocation problem by distributing the scheduled resources based on the agent interactions in the market. To explore the use of market mechanism for the coordination of distributed planning module in supply chain management, we have developed a prototype supply chain model in dynamic environment for specifying and simulating computational markets with circulative structure. It has been clarified that the proposed methodology facilitates sophisticated SCM under dynamic conditions.
\end{abstract}

Key words: supply chain management, market-oriented programming, dynamic system, multi-agent

\section{INTRODUCTION}

Many manufacturing operations are designed to maximize throughput and lower costs with little consideration for the impact on inventory levels and distribution capabilities. Purchasing contracts are often negotiated with very little information beyond historical buying patterns. The result of these factors is that there is not a single, integrated plan for the organization - there were as many plans as businesses. Clearly, there is a need for a mechanism through which these different functions can be integrated together. Supply chain management (SCM) is a strategy through which such an integration can be achieved (Goldratt, 1992) (Fisher, 1994). 
Companies, both manufacturing and service, are creators of value, not simply makers of products. SCM focuses on globalisation and information management tools which integrate procurement, operations, and logistics from raw materials to customer satisfaction. Future managers are prepared to add product value, increase quality, reduce costs, and increase profits by addressing the needs and performance of supplier relations, supplier selection, purchasing negotiations, and so on.

Solving product distribution problem in SCM presents particular challenges attributable to the distributed nature of the computation. Each business unit in SCM represents independent entities with conflicting and competing product requirements and may possess localised information relevant to their interests. To recognise this independence, we treat the business units as agents, ascribing each of them to autonomy to decide how to deploy resources under their control in service of their interests.

In this paper, a distributed product distribution method can be analysed according to how well it exhibits the following properties:

- Self-interested agents can make effective decisions with local information without knowing the private information and strategies of other agents.

- The method requires minimal communication overhead.

- Solutions don't waste resources. If there is some way to make some agents better off without harming others, it should be done. A solution that cannot be improved in this way is called Pareto optimal.

- The environment surrounding agents has dynamic nature.

Conventional straightforward distributed policies do not possess these properties. Assuming that a product distribution problem in SCM must be decentralised, market concept can provide several advantages as follows:

- Markets are naturally distributed and agents make their own decisions about how to bid based on the prices and their own utilities of the goods.

- Communication is limited to the exchange of bids and process between agents and the market mechanism.

In this paper we formulate $\mathrm{SCM}$ as a discrete resource allocation operation under dynamic environment, and demonstrate the applicability of economic approach to this framework by simulation experiments.

\section{BASIC CONCEPT}

\subsection{Product distribution problem}

Product distribution in SCM is generally proceeded by distributed autonomous dealings amongst business units. There generally exist several 
criteria in the business dealings, and we focus on principle elements, price and quantity, as a basic study in this paper.

Product distribution belongs to multi-objective optimisation problems, and severe conflicts between suppliers and demanders would occur due to the tradeoffs of their utilities. Market mechanism is expected to solve the problems by presenting a Pareto optimal solution for all of the business units in the market.

\subsection{Market metaphor and SCM}

Virtual market is a kind of computational market which consists of two types of heterogeneous agents, supplier and demander. Agent activities in terms of products required and supplied are defined so as to reduce an agent's decision problem to evaluate the tradeoffs of acquiring different products in market-oriented programming (Wellman, 1993). These tradeoffs are represented in terms of market prices, which define common scale of value across the various products. The problem for designers of computational markets is to specify the mechanism by which agent interactions determine prices.

In this paper the framework of general equilibrium theory (Okuno, 1985), which is proposed in microeconomics research field, has been adopted. In economics, the concept of a set of interrelated goods in balance is called general equilibrium. The general equilibrium theory guarantees a Pareto optimal solution in perfect competitive market. The connection between computation and general equilibrium is not all foreign to economists, who often appeal to the metaphor of market systems computing the activities of the agents involved. Some apply the concept more directly, employing computable general-equilibrium models to analyse the effects of policy options on a given economic system (Shoven, 1992). Obviously SCM model is well-structured for market-oriented programming, and that means the proposed concept takes advantage of the theory, and a Pareto optimal solution, which is conducted by microeconomics, is attainable in product distribution problem in SCM (Kaihara, 1999a).

\subsection{Market-Oriented programming}

Market-oriented programming is the general approach of deriving solutions to distributed resource allocation problems by computing the competitive equilibrium of an artificial economy (Kaihara, 1999b). It involves an iterative adjustment of prices based on the reactions of the agent 
in the market. Negotiation mechanism in market-oriented programming is shown in figure 1.

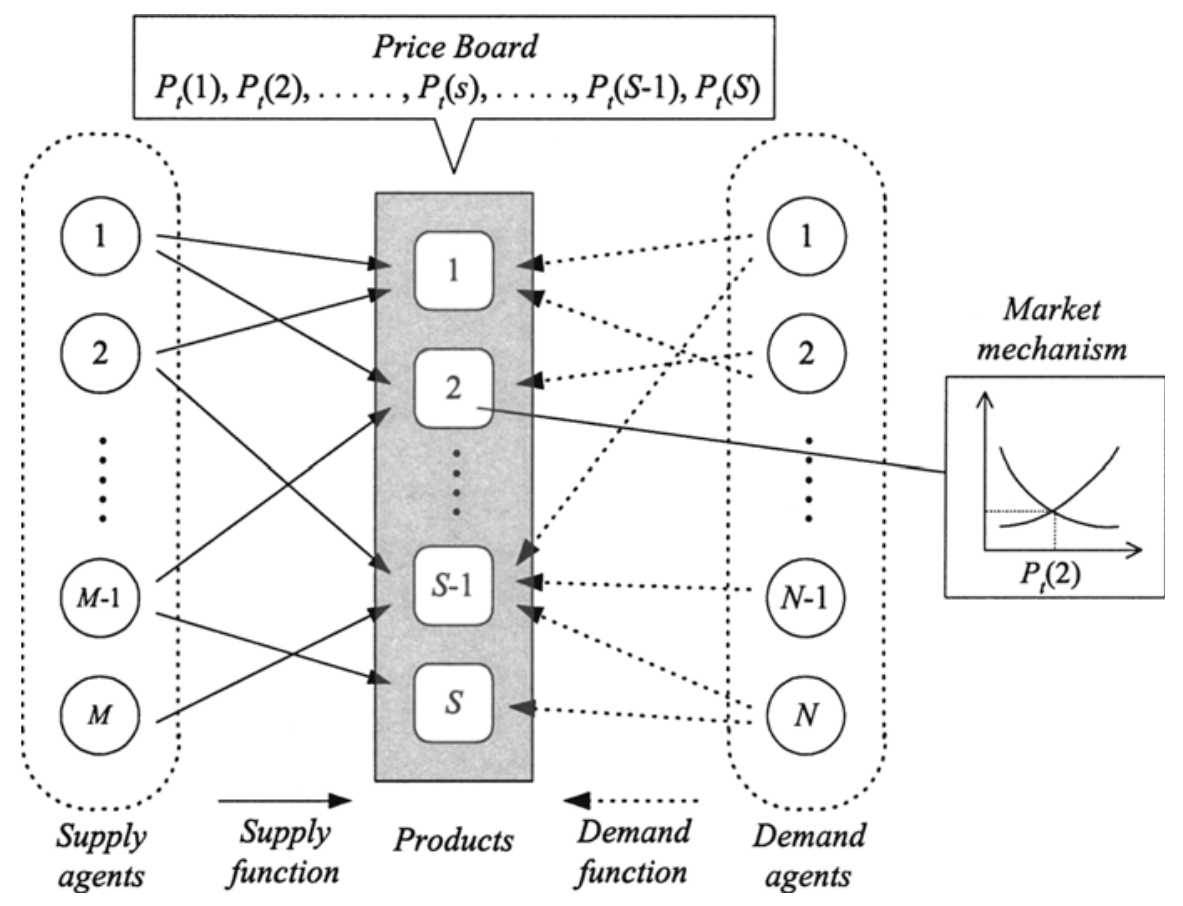

Figure 1. Virtual market structure

Let $P_{t}(s)$ be the price of resource $s$ at time $t$. Market mechanism computes an equilibrium price in each separate market. It involves an iterative adjustment of prices based on reactions of agents in the market. Product $s$ receives supply and demand functions and the market mechanism involved in the product adjusts individual prices to clear, rather than adjusting the entire price vector by some increment. The mechanism associates an auction with each distinct resource. Agents act in the market by submitting bids to auctions. In this paper bids specify a correspondence between prices and quantities of the resource that the agent offers to demand or supply as a basic study. Given bids from all interested agents, the auction derives a marketclearing price.

The algorithm of the proposed market-oriented programming in SCM is shown as follows:

Step 1: A supply agent $m$ sends bids to the market to indicate its willingness to sell the product $s$ according to its current price $P_{t}(s)$ 
in time $t$. The supply agent willingness is defined as a supply function in the bid message. The agent can send bids to the market within the limits of its current inventory level.

Step 2: A demand agent $n$ sends bids to a market to indicate its willingness to buy the product $s$ according to its current price $P_{t}(s)$ in time $t$. The demand agent willingness is defined as a demand function in the bid message. The agent can send bids to the market within the limits of its domestic budget. Each product has its own market, and they construct a competitive market mechanism as a whole.

Step 3: The market in product $s$ sums up supply functions and demand functions, then revises balanced price $P_{t}{ }^{\prime}(s)$ of product $s$ in time $t$. All the market must revise their balanced price via the same process.

Step 4: Check the balanced prices of all the products and if all the prices are fully converged, the acquired set of the prices is regarded as equilibrium price, then go to Step5. If not, go to Step 1.

Step 5: If dealing time is up, then stop. And if not, $t=t+1$ and go to Step 1.

Each agent maintains an agenda of bid tasks, specifying in which it must update its bid or compute a new one. The bidding process is highly distributed, in that each agent needs to communicate directly only with the auctions for the resources of interest. Each of these interaction concerns only a single resource; the auctions never coordinate with each other. Agents need not to negotiate directly with other agents, nor even know of each other's existence. As new bids are received at the auctions, the previously computed clearing price becomes obsolete. Periodically, each auction computes a new clearing price if any new or updated bids have been received, and posts it on the tote board. When a price is updated, this may invalidate some of an agent's outstanding bids, since these were computed under the assumption that price for remaining resources were fixed at previous value. On finding out about a price change, an agent argues its task agenda to include the potentially affected bids. At all times, the market-oriented mechanism maintains a vector of going prices and quantities that would be exchanged at those prices. While the agents have nonempty bid agendas or the auctions new bids, some or all resources may be in disequilibrium. When all auctions clear and all agendas are exhausted, however, the economy is in competitive equilibrium.

In the following chapter we clarify the heterogeneous agents definitions in dynamic environment, where trading conditions change dynamically during the task processing. 


\section{ECONOMIC AGENT}

\subsection{Production function}

Suppose supply agent $k$ has a production function $f_{k}$. In this paper we adopt a simple linear function as the production function described in equation (1).

$$
y_{k}=f_{k}\left(x_{k}\right)=a_{k} x_{k}
$$

where $x_{k}$ and $y_{k}$ are the amount of input / output resources of agent $k$, respectively, and $a_{k}$ is a constant value that represents productivity.

The relation between the amount of input materials $i\left(x_{k i}\right)$ and the cost $\left(c_{k}\right)$ is the other important factor in SCM, and we define the following function based on logistic curve. The reason of applying the logistic curve is that we have a limitation of production due to resource constraints, in other words, diminishing returns.

Then we have

$$
\frac{d x_{k i}}{d t}=h_{k}\left(1-\frac{c_{k}}{r_{k}}\right) \times c_{k}
$$

$$
x_{k i t}=\int^{+1} d x_{k i}=h_{k}\left(1-\frac{c_{k t}}{r_{k}}\right) \times c_{k t}
$$

where $x_{k i t}$ and $c_{k t}$ are the amount of input material $i$ and its cost of agent $k$ at time $t$, respectively.

\subsection{Cost function}

The turnover of agent $k\left(w_{k}\right)$ by selling product $j$ is shown in equation (4).

$$
w_{k}=q_{j} s_{k j}
$$

where $q_{j}$ and $s_{k j}$ are the selling price and the total amount of supplying product $j$ in agent $k$, respectively.

The total cost of agent $k\left(v_{k}\right)$ is

$$
v_{k}=p_{i} d_{k i}+c_{k}
$$

where $p_{i}$ and $d_{k i}$ are the buying price and the total amount of demand product $i$ in agent $k$, respectively. Then finally cost function is defined as follows:

$$
w_{k}=q_{j} s_{k j}-p_{i} d_{k i}-c_{k}
$$




\subsection{Commerce in virtual market}

All the agents in the virtual market try to get the maximised profit in their trading. The basic principle of the agent is that it requires just the amount of materials for its production at this moment.

It requires processing time for manufacturing, and the trading conditions should be changed during the manufacturing process in dynamic environment. The objective function $\left(u_{k d t}\right)$ for demand of agent $k$ at time $t$ is

$$
u_{k d t}=\left(a_{k} q_{j t}-p_{i t}\right) h_{k}\left(1-\frac{c_{k t}}{r_{k}}\right) c_{k t}-c_{k t}
$$

where $p_{i t}$ and $q_{j t}$ are the demand price and supply price at time $t$, respectively. Then the demand $\left(d_{k i t}\right)$ of agent $k$ at time $t$ is attainable as the maximum value of $u_{k d t}$. Since the logistic curve is a concave down function, $u_{k d t}$ is maximised to substitute the following $c_{k t}$ for equation (3):

$$
\begin{cases}\left(a_{k} q_{j t}-p_{i t}\right) h_{k}\left(1-\frac{2 c_{k t}}{r_{k}}\right)-1=0 & \left(\text { for }\left(a_{k} q_{j t}-p_{i t}\right)>0\right) \\ c_{k t}=0 & \left(\text { for }\left(a_{k} q_{j t}-p_{i t}\right) \leq 0\right)\end{cases}
$$

The objective function $\left(u_{k s t}\right)$ for supply of agent $k$ at time $t$ is

where $\quad s_{k j t}=a_{k} h_{k}\left(1-\frac{c_{k t}}{r_{k}}\right) c_{k t}$

$$
u_{k s t}=q_{j t} s_{k j t}-p_{i(t-1)} \frac{1}{a_{k}} s_{k j t}-c_{k t}
$$

and the amount of supply $\left(s_{k i t}\right)$ of agent $k$ at time $t$ is attainable as the maximum value of $u_{k s t}$. Then $u_{k s t}$ is maximised to substitute the following $c_{k t}$ for equation (10):

$$
\begin{cases}\left(a_{k} q_{j t}-p_{i(t-1)}\right) h_{k}\left(1-\frac{2 c_{k t}}{r_{k}}\right)-1=0 & \left(\text { for }\left(a_{k} q_{j t}-p_{i(t-1)}\right)>0\right) \\ c_{k t}=0 & \left(\text { for }\left(a_{k} q_{j t}-p_{i(t-1)}\right) \leq 0\right)\end{cases}
$$

\subsection{Agent optimisation strategy under budget constraint}

All the agents have budget constraint so as to realise our market model in SCM. They naturally try to maximise their profit in their economic behaviours under the constraint. Suppose the total expense for resource set $l$ to produce $j$ from $i$ in agent $k$ at time $t$ is $c_{k l, t}$, and the maximum budget is $e_{k}$, then we have 


$$
\sum_{l} c_{k l, t} \leq e_{k}
$$

Agents naturally try to maximise their individual utility and we can adopt Profit Maximise Theorem (kaihara, 1999c) as the agents' strategy. The Profit Maximise Theorem requires two types of preconditions, such as:

i) $f_{k}$ (production function) is differentiable

ii) $\left.\forall l \mathrm{~F} \frac{\partial f_{k l}}{\partial x_{k l}}\right|_{x_{k l}=x}>\left.\frac{\partial f_{k l}}{\partial x_{k l}}\right|_{x_{k l}=x+\Delta}$

and these conditions are obviously satisfied by equation (1),(3) in this paper. The theorem endorses that agent $k$ maximises its profit to calculate the minimised $m_{k}$, which satisfies the following equation (13):

$$
\forall l \mathrm{~F} \frac{\partial u_{k d}}{\partial v_{k l}}=m_{k}\left(m_{k} \geq 0\right) \quad \bigcap \quad v_{k} \leq e_{k}
$$

Finally we can get $x_{k l t}$, which maximises the function $u_{k d l}$, by substituting $c_{k l}$ acquired from equation (14) for equation (3).

$$
\frac{\partial u_{k d l}}{\partial c_{k l}}=\left(a_{k l} q_{i}-p_{i}\right) h_{k l}\left(1-\frac{2 c_{k l}}{r_{k l}}\right)-1=m_{k}
$$

\section{COMPUTER SIMULATION}

\subsection{Experimental circulative SCM model}

A primitive product life cycle SCM model, shown in figure 2, was developed to investigate the validity of the proposed approach. As a basic research, the experimental model is quite simple enough to evaluate the basic dynamism of the agent interactions, defined in the previous chapter.

There exist two agents, which construct a circulatory resource flow via two markets, and this model represents the simplest structure of real supply flows. The agents would affect themselves with some time delay in the model. Each market has outside supply / demand, which are defined as primitive Cobb-Douglas function (Okuno, 1985) shown in equation (15).

$$
y_{i}=a_{j} p^{b_{j}}
$$


The experimental parameter settings are as follows:

Agent: $a_{k}=1.2, h_{k}=5.0, r_{k}=100, e_{k, 0}=10, z_{k j, 0}=10$

Outside supply function: $a_{j}=0.1, b_{j}=1.2$

Outside demand function: $a j=10.0, b_{j}=-1.0$

Market: $p_{j, 0}=1.0, q_{j, 0}=1.0, k=1,2$

where ${ }_{k, 0}$ and ${ }_{j, 0}$ mean initial value of agent $k$ and resource $j$, respectively.

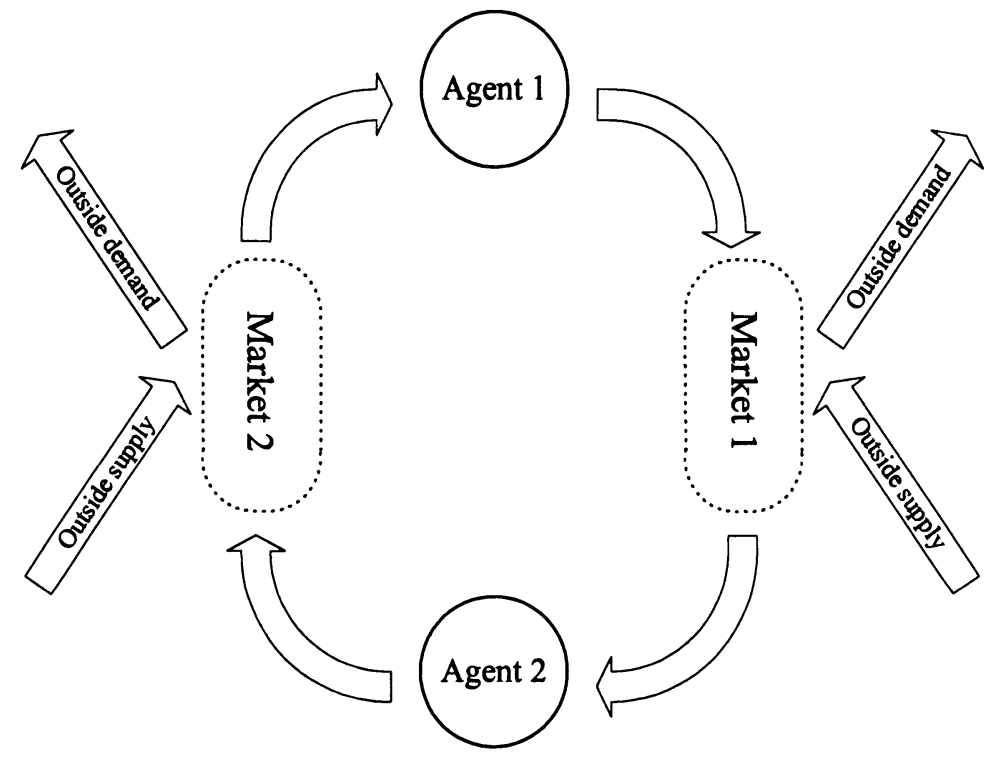

Figure 2. Primitive product life cycle SCM model

\subsection{Basic dynamism}

Basic dynamism of resource allocations in terms of financial items (budget and price) and stock (product) is shown in figure 3.

Initially $(t<4)$ agents try to hold down their production and sell their stocks due to cheap selling prices. Then the over demand situation appears, and that causes the increase of the selling prices $(t<6)$. Finally the markets are gradually converged into the equilibrium prices endorsed by microeconomics.

Since the model has circulatory structure, initial resource prices should affect the system dynamism obviously. Figure 4 illustrates the simulation results in $\left(p_{j, 0}=1.0, q_{j, 0}=2.5\right)$. The result with the vibrated curves is completely different from figure 3 . It is quite interesting to confirm that the 
small initial price difference causes strong influence to the markets due to a dynamic interaction and two-way enrichment process between micro and macro processes of change.

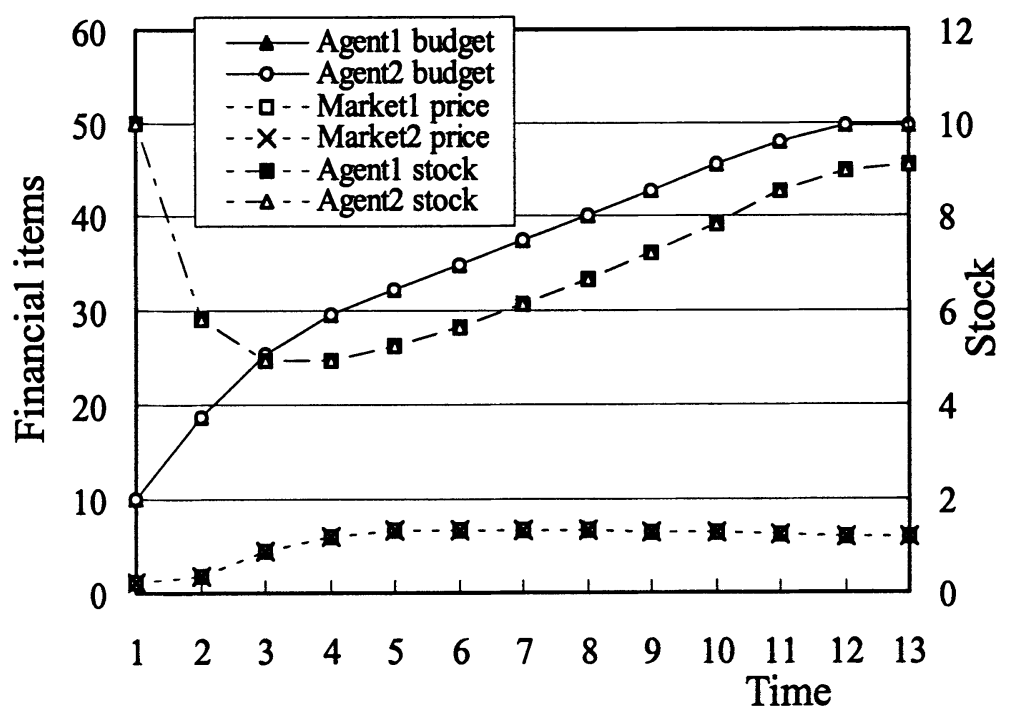

Figure 3. Basic dynamism $\left(p_{j, 0}=1.0, q_{j, 0}=1.0\right)$

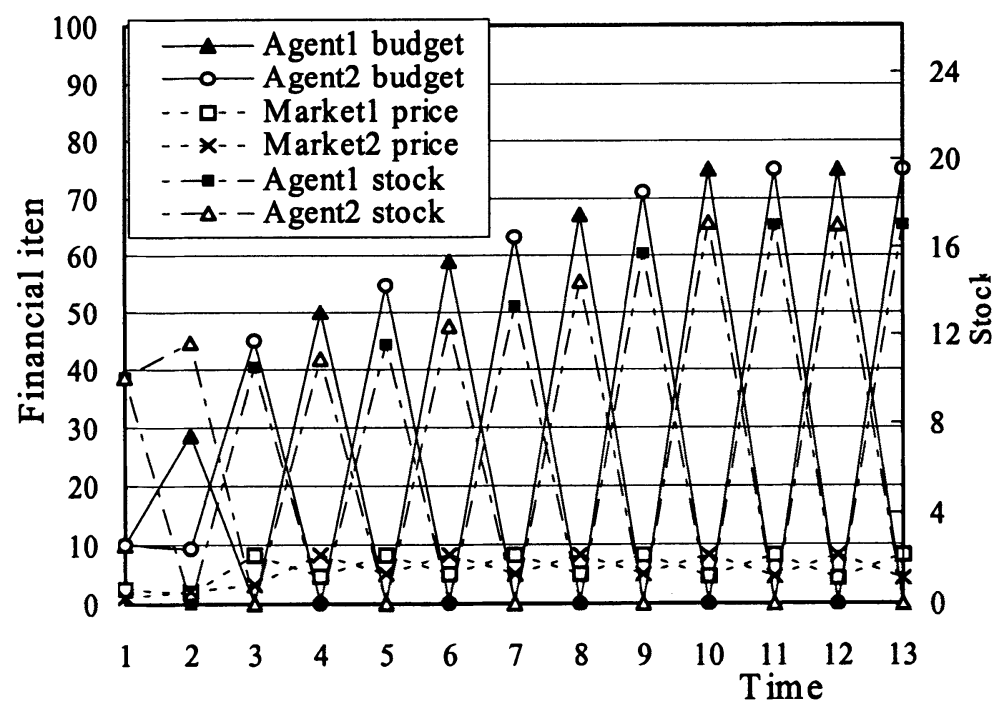

Figure 4. Basic dynamism $\left(p_{j, 0}=1.0, q_{j, 0}=2.5\right)$ 


\subsection{Productivity effects}

Constant parameter $a_{k}$ in equation (1) is regarded as productivity in the agent definition. The experimental results on the productivity are shown in figure 5 . The agents productivity $\left(a_{k}\right)$ was set to 2.0 in this figure.

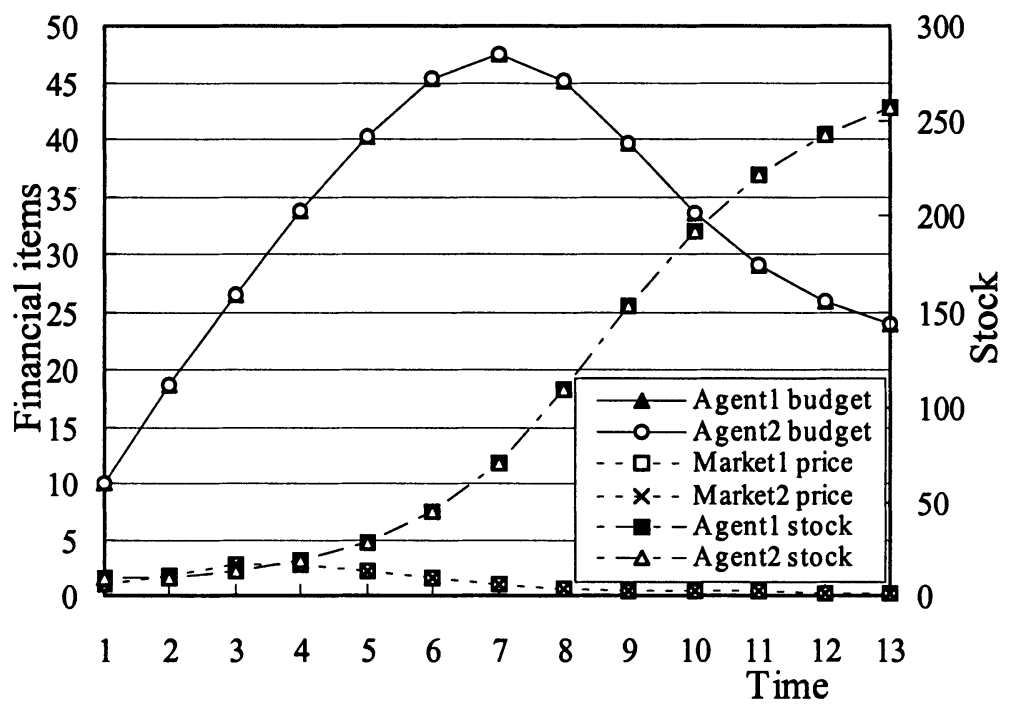

Figure 5. Productivity effects

Generally it is well known that the rapid increase of total productivity leads to excessive competitions in the market. Product prices decrease there, and that causes financial difficulties in all the business units. We can often observe it in semiconductor production industry as an example.

It is quite interesting to have confirmed that the experimental results in figure 5 have some analogy to the general market phenomenon. It is obvious that the rapid productivity increase is short-sighted and makeshift in the resource-oriented ceiling market. Formulating the agent strategy with dynamic utilities enables us to handle the transitional trends with reality.

\section{CONCLUSIONS}

In this paper SCM was formulated as a discrete resource allocation problem with dynamic environment, and the several experimental results demonstrated the applicability of economic analogy to this framework. It has been confirmed that careful constructions of the decision process according to economic principles can lead to efficient distributed resource allocation in 
$\mathrm{SCM}$, and the dynamic behaviour of the system can be analysed in economic terms.

\section{Acknowledgements}

This research has been supported by IMS international research program in Japan, under contract No.0019 (HUTOP project).

\section{REFERENCES}

1. Fisher ML, Hammond JH, Making supply meet demand in uncertain world, Harvard Business Review, May/Jun, 1994.

2. Goldratt EM, The GOAL, North River Press, 1992.

3. Kaihara T, Supply Chain Management with Multi-agent Paradigm, Proceedings of the 8th IEEE International Workshop on Robot and Human Interaction, pp394-399, 1999a.

4. Kaihara T., Supply Chain Management based on Market Mechanism in Virtual Enterprise, Infrastructures for Virtual Enterprises, Kluwer Academic Publishers, Boston, pp.399-408, 1999b.

5. Kaihara T., Supply Chain Management with Market Economics, Manufacturing for a global market, M. T. Hillery \& H. J. Lewis Eds., Vol. 1, pp.659-662, 1999c.

6. Okuno H, Suzumura K, Micro economics I, Iwanami, 1985.

7. Shoven JB, Whalley J, Applying General Equilibrium, Cambridge University Press, 1992.

8. Wellman MP, A Market-Oriented Programming Environment and its Application to Distributed Multi- commodity Flow Problems, ICMAS96, 385-392, 1996.

\section{BIOGRAPHY}

Toshiya Kaihara received the B.E. and M.E. degrees in precision engineering from Kyoto University, Kyoto, Japan, in 1983 and 1985, respectively. He received the Ph.D. degree in mechanical engineering from Imperial College, University of London, London, UK, in 1994. He is currently an associate professor of Computer and Systems Engineering at Kobe University. $\mathrm{He}$ is a member of Japan Society for Precision Engineering, IEEE, IFIP and many others. 